\title{
Spectral imaging: Towards Quantitative X-ray Microanalysis
}

\author{
P.G. Kotula and M.R. Keenan
}

\author{
Sandia National Laboratories, PO Box 5800, Albuquerque, NM 87185-0886
}

Spectral imaging, where a series of complete x-ray spectra are collected from a 2D area, holds great promise for comprehensive microanalysis. The challenge remains, however, to acquire enough data and robustly analyze it all. Spectral images come in a range of sizes but 'small' ones typically comprise 64 by 64 pixels (4096 spectra) or 128 by 128 pixels (16384 spectra). In terms of typical SEM images, these are decidedly low-resolution. A prime goal of utilizing spectral images in routine and comprehensive microanalysis is therefore to surpass 1000 by 1000 -pixel $\left(10^{6}\right.$ spectra $)$ spectral images. Automated multivariate statistical analysis (MSA) methods have been applied successfully to spectral images up to 128 by 128 pixels as described previously [1]. In the present paper we describe the extension of that work to much larger data sets.

As spectral images increase in size, manual analysis methods quickly become cumbersome. Additionally they are typically subjective and may require that the individual spectra in the spectral image contain a large number of counts [2]. Optimized MSA methods have been shown to be robust and capable of handling very noisy data [1] because statistically similar pixels are grouped together to produce a 'super spectrum' with the collective statistics. MSA techniques, where the energy channels (one thousand or more) are considered the variables, have been considered viable so long as the entire analysis is not bigger than the amount of free memory (RAM) on the computer. For 128, 256, 512 and 1024 pixel $^{2}$ (by 1024 energy channels) calculations a minimum of 135, 537, 2147, and 8590 Mbytes of free memory, respectively, would be required (assuming that the calculation does not require appreciably more space). We have developed an efficient method for automating the MSA algorithm in cases where the data set is too large to fit in memory. The method is based on spectral unmixing of pixel neighborhoods and retains full spectral and spatial resolution in the final spectral and component map estimates. To demonstrate this, a 512 by 512 pixel by 1024 energy-channel spectral image was acquired from a geologic material (described in more detail elsewhere [3]). 64x64 arrays of 8x8 pixel neighborhoods were formed from the original data set and then optimized MSA calculations were performed on this reduced-size data set. The results of this calculation (i.e., the 'pure' components) were then fit to the original full-resolution raw data. The data was acquired on a JEOL 5900LV SEM operating at 20kV equipped with a NORAN Vantage (Digital Imaging with Spectral Imaging), $2 \mu \mathrm{m}$ /pixel (1 $\mathrm{mm}$ field of view), a $9 \mu \mathrm{sec}$ pulse-processor shaping time, $9 \mathrm{msec}$ total dwell time per pixel, and $40 \%$ dead time resulting in an average of 100 counts per spectrum. The entire calculation took 3 minutes, resulting in 15 components. The results for 128 by 128 arrays of 4-pixel neighborhoods on the same data set were virtually indistinguishable from the 64 by 64 arrays of 8-pixel neighborhoods case, differing only by a few minutes in the amount of time to calculate the solution. Figure 1 shows component images and spectra from $\mathrm{S}, \mathrm{Pb}, \mathrm{Fe}$ and $\mathrm{Cu}$ delineating the different sulfides present in the specimen. Because $\mathrm{S}$ is present in three different phases ( $\mathrm{Fe}-\mathrm{S}, \mathrm{Cu}-\mathrm{S}$ and $\mathrm{Pb}-\mathrm{S})$ and $\mathrm{Pb}$ is present in two additional phases ( $\mathrm{Pb}-\mathrm{Te}$ and $\mathrm{Pb}-\mathrm{Te}-\mathrm{O}[3]$ ), there will not be distinct 'phase' images and spectra and instead almost pure elemental images and spectra are observed [1]. Figure 2 is an overlay of the reconstructed spectrum from $\mathrm{Pb}-\mathrm{S}$, and $\mathrm{Pb}$ - and $\mathrm{S}$-components for one pixel of $\mathrm{Pb}-\mathrm{S}$. The reconstructed spectrum represents the sum of the products 
of all the component images and spectra, not just $\mathrm{Pb}$ and $\mathrm{S}$. Only $\mathrm{Pb}$ and $\mathrm{S}$ components contribute appreciably to the $\mathrm{Pb}-\mathrm{S}$ reconstructed spectrum though.

\section{References}

[1] P.G. Kotula et al., Microsc. Microanal. In press (2002).

[2] P.G. Kotula and M.R. Keenan, These proceedings (2002).

[3] P. G. Kotula, P.F. Hlava, and M.R. Keenan, These proceedings (2002).

[4] Sandia is a multiprogram laboratory operated by Sandia Corporation, a Lockheed Martin Company, for the United Stated Department of Energy (DOE) under contract DE-AC04-94AL85000.
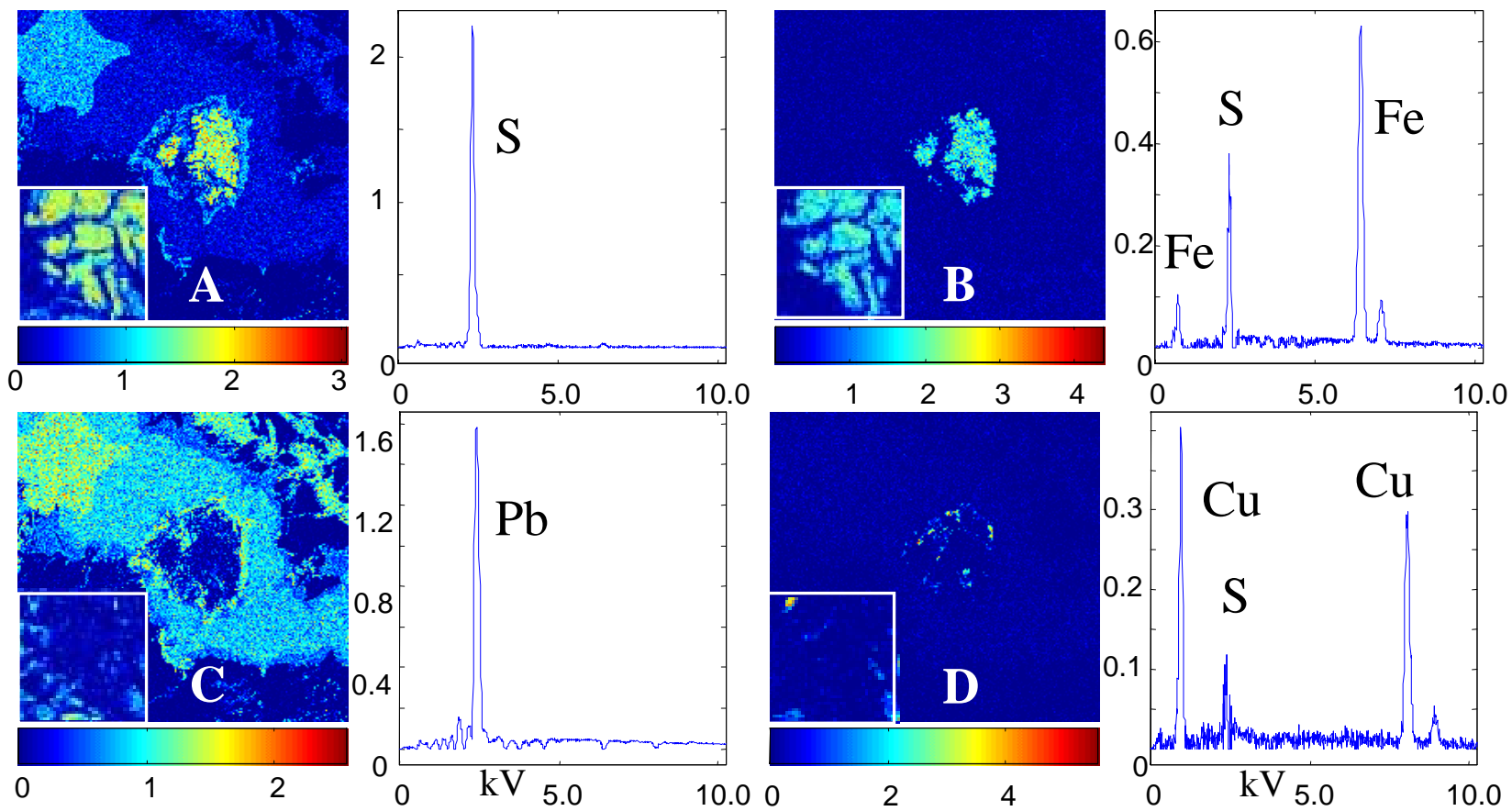

FIG. 1. Four 'pure' component images and spectra corresponding to: A. S; B. Fe-S; C. Pb and D. Cu$\mathrm{S}$. Spectral intensities are in normalized units. Inset are enlarged regions from the center.

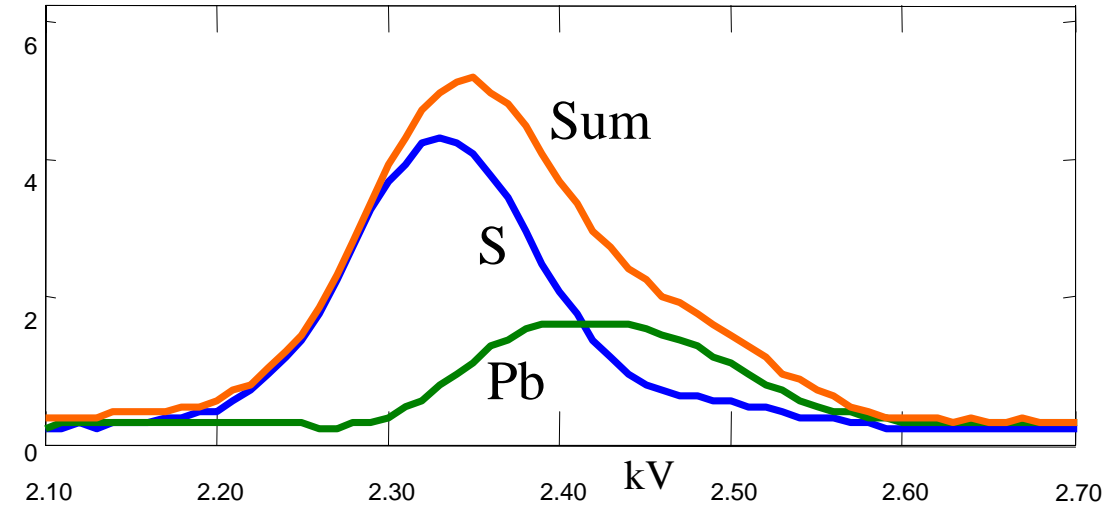

FIG. 2. Pure component spectra from $\mathrm{Pb}$ and $\mathrm{S}$ and the corresponding superposition of all pure components for one pixel from the $\mathrm{Pb}-\mathrm{S}$. 